\title{
Liver tissueoid composed of electrospun multiscale fiber enhance hepatic differentiation and therapeutic function of chemical derived hepatic stem cells
}

\author{
Yohan Kim, Sangtae Yoon, Da Hee Hong, Myoung-Hoi Kim, Taehun Kim, Jaemin Jeong, Dongho Choi
}

Division of Hepatobiliary, Department of Surgery, Hanyang University College of Medicine, Seoul, Korea

Background: Artificial tissueoid has tissue-like properties that consist of multicellular component and three-dimension microenvironments. This model recapitulated the in situ environment interactions by providing structure, physiology, and arrangement of individual cells. Here we introduce a novel artificial liver tissueoid platform which can provide in vivo liver multicellular microenvironments and modulate hepatocytes behavior.

Methods: The liver tissueoid was hierarchically assembled with cell-laden sheets of which base structures were composed of extracellular matrix (ECM) mimicking electrospun fiber mats. To generate liver tissueoid, human chemically-derived hepatic progenitors (hCdHs) are generated by small molecules as our previously reported. The microfabricated edge-framework for the fiber mat enabled not only stable culture of the functionalized cells but also multilayering construction of cell-laden complexes even including heterogeneous composition of assembly.

Results: The heterogenous multilayer stacking, assembling multi-layer stacking with both hCdHs and endothelial cells (HUVECs), significantly enhanced the functional properties of hCdHs to differentiate into hepatocytes and affect survival of mice in acute injury model. Especially, the differentiation potential of $\mathrm{hCdHs}$ was associated with OSM downstream signaling pathways. Interestingly, single layered liver tissueoid without HUVECs additionally more activated OSM-downstream signaling pathways, whereas double and triple layered liver tissueoid with HUVECs included both OSM-dependent and OSM-independent pathway which was mediated by selective activation of AKT signaling.

Conclusions: Overall, our results suggested that liver tissueoid showed efficient hepatic differentiation capacity of hCdHs and exhibited therapeutic effect after transplantation into liver injury mouse. 